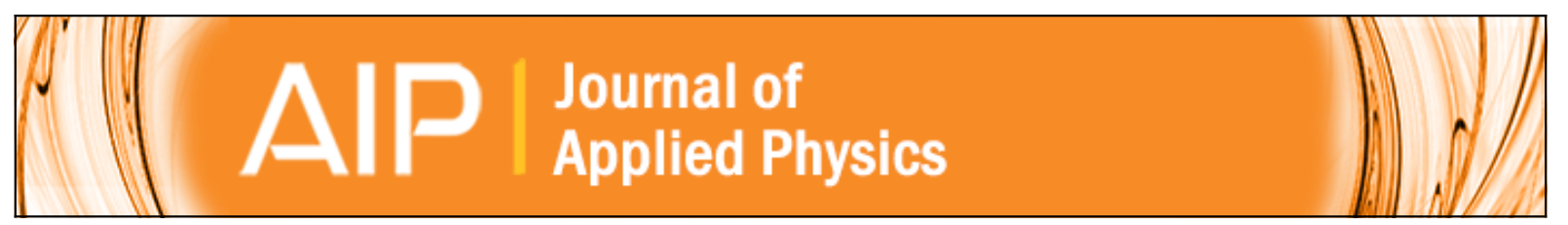

Microwave eddy-current shielding effect in metallic films and periodic nanostructures of sub-skin-depth thicknesses and its impact on stripline ferromagnetic resonance spectroscopy

Ivan S. Maksymov and Mikhail Kostylev

Citation: Journal of Applied Physics 116, 173905 (2014); doi: 10.1063/1.4900999

View online: http://dx.doi.org/10.1063/1.4900999

View Table of Contents: http://scitation.aip.org/content/aip/journal/jap/116/17?ver=pdfcov

Published by the AIP Publishing

Articles you may be interested in

Eddy-current effects on ferromagnetic resonance: Spin wave excitations and microwave screening effects

J. Appl. Phys. 119, 163903 (2016); 10.1063/1.4948302

Coupling of microwave magnetic dynamics in thin ferromagnetic films to stripline transducers in the geometry of the broadband stripline ferromagnetic resonance

J. Appl. Phys. 119, 013901 (2016); 10.1063/1.4939470

Influence of the interface on the magnetic properties of ferromagnetic ultrathin films with various adjacent copper thicknesses

J. Appl. Phys. 115, 17C108 (2014); 10.1063/1.4861555

Ferromagnetic resonance study of polycrystalline cobalt ultrathin films

J. Appl. Phys. 99, 08 N503 (2006); 10.1063/1.2151832

Effect of conformal roughness on ferromagnetic resonance linewidth in thin Permalloy films

J. Appl. Phys. 97, 10A721 (2005); 10.1063/1.1860271

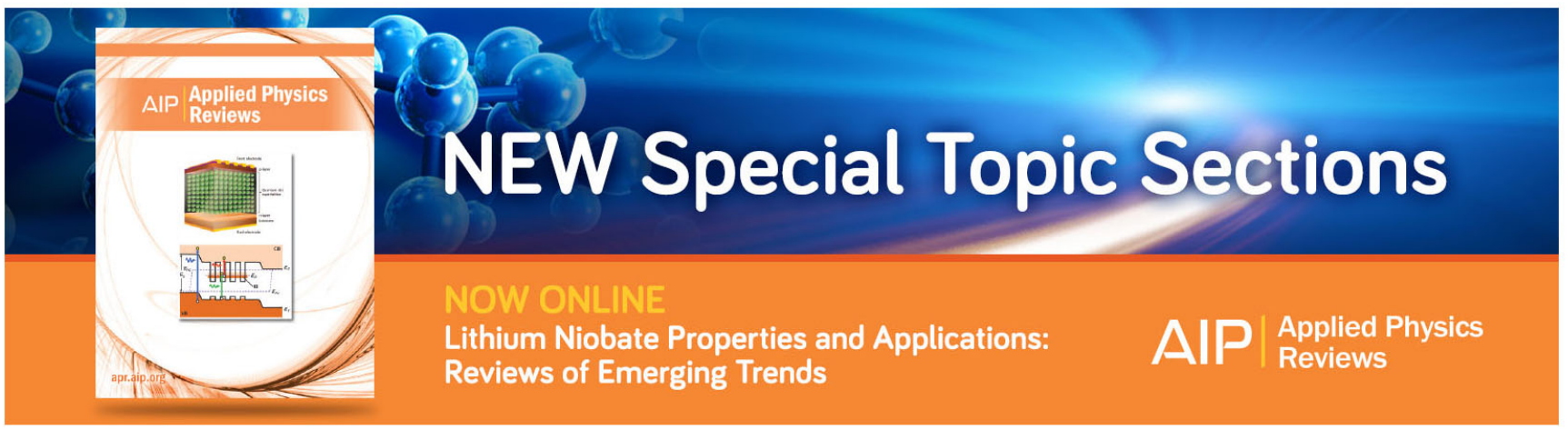




\title{
Microwave eddy-current shielding effect in metallic films and periodic nanostructures of sub-skin-depth thicknesses and its impact on stripline ferromagnetic resonance spectroscopy
}

\author{
Ivan S. Maksymov and Mikhail Kostylev \\ School of Physics, M013, The University of Western Australia, Crawley, WA 6009, Australia
}

(Received 13 August 2014; accepted 22 October 2014; published online 4 November 2014)

\begin{abstract}
A strong microwave shielding effect due to the excitation of microwave eddy-currents exists for metallic films of sub-skin-depth thickness $(10-100 \mathrm{~nm})$. If the film is ferromagnetic, this effect strongly influences results of the broadband stripline ferromagnetic resonance (FMR) spectroscopy. It also potentially hampers the development of magnetically tuneable metamaterials. By means of rigorous numerical simulations, we address an important problem of the dependence of the eddy current effect on the width of the stripline used for driving magnetisation dynamics in the broadband FMR spectroscopy. We study theoretically electrodynamics of realistic striplines and also extend the main result from the case of continuous conductive films to periodic conductive nanostructures-magnonic crystals. Based on these findings, we also give recommendations on improving performance of magnetically tuneable metamaterials, which are based on conductive ferromagnetic films and nanostructures. In our simulations, we consider examples of microstrip lines which are $5 \mu \mathrm{m}$ to $1.5 \mathrm{~mm}$ wide. However, the simulation results should be equally applicable to coplanar waveguides with the same width of the signal line. (C) 2014 AIP Publishing LLC.

[http://dx.doi.org/10.1063/1.4900999]
\end{abstract}

\section{INTRODUCTION}

Electric- and magnetic-field shields play more and more active role in physics of operation of integrated circuits, ${ }^{1}$ touch screens, ${ }^{2}$ and many other electronic devices. ${ }^{3}$ For example, interconnections and passive components surrounding the active devices of integrated circuits must be perfectly shielded from semiconducting silicon substrates, because the latter possesses considerable electrical conductivity and causes losses in passive components. ${ }^{1}$ Whereas conductive thin films and periodic structures act as electricfield shields, magnetic-field shields are created by eddy currents induced in conductive materials by microwave magnetic fields.

In the field of microwave spintronics and magnonics, ${ }^{4}$ which is highlighted as a future emerging research directions in modern magnetism, ${ }^{5}$ microwave eddy-current shielding effects may play an important role. ${ }^{6,7}$ Spin waves are eigenexcitations in ferromagnetic materials, existing in the $\mathrm{GHz}$ frequency range. Spin waves represent collective precessional motion of electron spins. Ferromagnetic resonance (FMR) is a special case of spin waves where all the spins precess in phase over the whole volume of a magnetic material.

Most magnonic and metal spintronic nanodevices rely on thin (5-100 $\mathrm{nm}$ or even thinner) continuous and periodically nano-patterned film materials made from ferromagnetic metals (see, e.g., Refs. 8-10). The ferromagnetic metal films (e.g., $\mathrm{Fe}, \mathrm{Co}, \mathrm{Ni}, \mathrm{Ni}_{80} \mathrm{Fe}_{20}$, etc.) possess high electrical conductivity. Their microwave response is often probed with the broadband stripline ferromagnetic resonance (BSFMR) spectroscopy. ${ }^{8,10-16}$ BSFMR is well suited for characterising magnetic properties of ferromagnetic films and planar nanostructures, and for studying their physics. ${ }^{17,18}$
A BSFMR arrangement is also used to excite magnetically tuneable metamaterials, which consist, e.g., of a large array of split-ring resonators excited by the microwave field of the microstrip line and coupled to a magnetic film or nanostructure. ${ }^{19-21}$ Metamaterials are a rich research field motivated by the promise of both ground-breaking science and device applications based on a negative index of refraction $^{22}$ and cloaking at microwave frequencies. ${ }^{23}$ Recent works on this topic ${ }^{20,21}$ have revealed an adverse effect of the eddy current shielding in BSFMR-based metamaterials exploiting continuous ferromagnetic metal films. To circumvent this effect, it has been recommended to use either magneto-insulating materials (e.g., $\mathrm{YIG}=$ Yttrium Iron Garnet ${ }^{20}$ or micro-patterned FM films ${ }^{21}$ instead of continuous ones.

In a typical BSFMR measurement, the ferromagnetic metal sample is placed on top of a microwave microstrip or coplanar stripline, and the FMR absorption is measured with a vector network analyser, which also serves as a source of microwave current. This current flows through the stripline and creates a microwave dynamic magnetic field around it. This field excites dynamic magnetisation in the ferromagnetic metal film sitting on top of the stripline. Peaks in the transmission spectrum of the stripline appear due to absorption of microwave power at the frequencies of excitation of the fundamental mode of quasi-uniform precession of magnetisation and of higher-order standing spin wave modes (SSWMs) across the film thickness. ${ }^{6,8}$

Shielding of the driving microwave field by eddy currents in highly conductive ferromagnetic films strongly affects BSFMR results because of the important peculiarity of the stripline FMR: the single-surface incidence of the microwave magnetic field on the film. In the previous works, 
Refs. 6-8, 24, and 25, it was demonstrated, experimentally and theoretically, that a sub-skin-depth-thick metallic film acts as a perfect shield for a microwave magnetic field created by wide (e.g., $0.3 \mathrm{~mm}$-wide) microstrip lines. In the case of perfect shielding, the microwave field amplitude inside the film decays almost linearly from its maximum at one film surface to zero at the other film surface.

This quasi-linear decay of the dynamic magnetic field inside the film has important implications. It is known that the perfect symmetry/anti-symmetry of the SSWMs makes them unobservable while driven by a spatially uniform microwave magnetic field typical for an FMR setup. However, these modes become driven in the presence of the highly non-uniform quasi-linear profile of the microwave magnetic field inside the ferromagnetic metal film. ${ }^{6,8,24}$

In Ref. 8, it has also been predicted that the shielding of the microwave field may be reduced or even vanish in BSFMR configurations with narrow (e.g., $30 \mu \mathrm{m}$ wide) striplines often used in recent experimental works (see, e.g., Refs. 26 and 27). This prediction is supported by results of rigorous semi-analytical Bubnov-Galerkin calculations and numerical finite-difference time-domain (FDTD) simulations of dispersion characteristics of surface spin waves in ferromagnetic metal thin films. ${ }^{25}$ In BSFMR experiments employing narrow microstrip lines, surface spin waves with non-zero wave vectors values up to $k_{\max }=2 \pi / w$ are exited (where $w$ is the width of the microstrip line or the characteristic width of the coplanar waveguide). Calculations of the eigen-profiles of the microwave magnetic field of spin waves (in the absence of excitation by microwave currents) show that for non-negligible $k$-values, the field becomes nearly uniform across the film thickness. This suggests that the incident microwave field of the stripline BFMR transducer may gradually become unshielded ${ }^{25}$ with an increase in $k$.

On the other hand, Bailleul ${ }^{7}$ has recently demonstrated perfect shielding of microwave magnetic field by a thin nonmagnetic metallic film sitting on top of a coplanar waveguide with a $350 \mu \mathrm{m}$-wide central conductor. Similar to the Bubnov-Galerkin approach used in Ref. 25, an eigenvalue problem was solved in Ref. 7 to obtain the result for the coplanar line, i.e., a source of a microwave current was not included in the calculation. Consequently, the dependence of the shielding effects on the source geometry-i.e., the stripline width-still remains unclear. Furthermore, a recent experiment on highly conductive periodic nanostructures has revealed a crucial effect of eddy currents on the excitation of SSWMs. ${ }^{28}$ Thus, one more question arises: whether the shielding in nanostructures with a high conductivity of metals is as perfect as in metallic continuous thin films?

In this work, we investigate, analytically and numerically, the following experimental situations of eddy-current shielding in (i) sub-skin-depth-thick conductive films excited by a microstrip line of a finite width $w=30-300 \mu \mathrm{m}$; and (ii) periodic structures consisting of conductive nanostripes of sub-skindepth thickness excited by an infinitely wide microstrip line. ${ }^{29}$ For simplicity, we consider that both thin films and nanostructures are made from a non-magnetic metal. As shown in Ref. 28, this approximation is able to deliver results which are in qualitative agreement with a BSFMR experiment.
In the first case, we demonstrate that the out-of-plane component of microwave magnetic field localised at the edges of narrow striplines is not shielded. This field passes through the film and forms closed loops by gradually transforming itself into the in-plane component in the space behind the film. Hence, the shielding by eddy currents becomes imperfect. Consequently, the non-uniformity of the microwave magnetic field inside the film becomes less pronounced. Importantly, the strength of the amplitude of the out-of-plane field component is a strong function of the stripline width. This leads to qualitatively different conditions for excitation of magnetisation dynamics by "narrow" and "wide" stripline transducers. ${ }^{6,8,24}$

In the second case, we demonstrate that the microwave magnetic field is not perfectly shielded by conductive nanostructures in the near field regime. In fact, the microwave magnetic field leaks through the air slits of the nanostructure and forms an evanescent field behind the nanostructure in its closest vicinity. Thus, the shielding becomes perfect first in the far-field zone.

\section{SHIELDING BY CONTINUOUS CONDUCTIVE FILMS}

The inset in Fig. 1 schematically shows the four investigated configurations in which a conductive thin film sits on top of a microstrip line of width $w$. The thickness of the film is $L=100 \mathrm{~nm}$ in all cases. The electrical conductivity of the film $\sigma=4.5 \times 10^{6} \mathrm{~S} / \mathrm{m}$ corresponds to the standard conductivity of permalloy $\left(\mathrm{Ni}_{80} \mathrm{Fe}_{20}\right)$, a metallic material largely

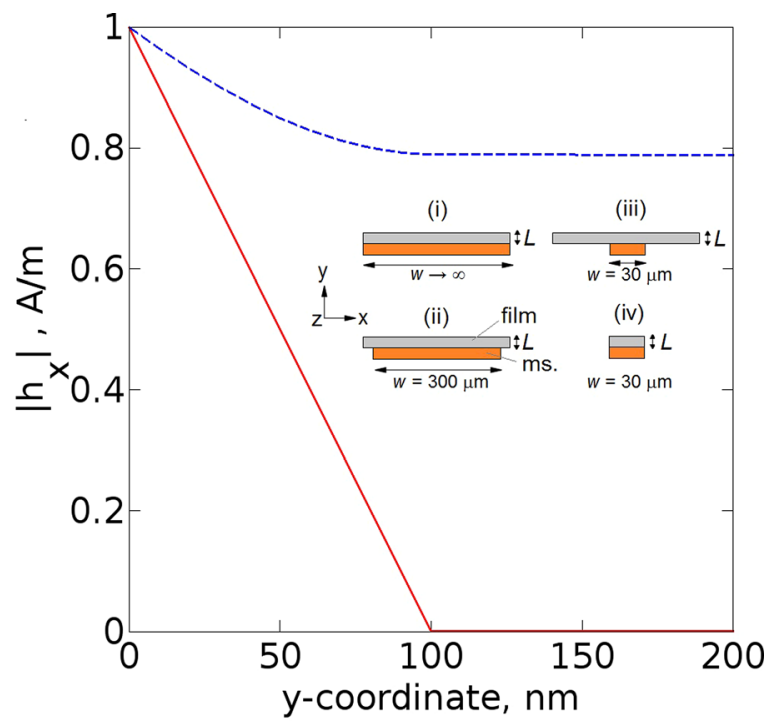

FIG. 1. Inset: Configurations of the microstrip lines ("ms.", bottom layer) with a conductive thin film sample on top ("film," top layer). The thickness of the film is $L=100 \mathrm{~nm}$ in all cases. The width $w$ of the microstrip line is assumed to be infinite in (i), $300 \mu \mathrm{m}$ in (ii), and $30 \mu \mathrm{m}$ in (iii) and (iv). The microwave current in the microstrip line flows in the $z$-direction (towards the reader). Both the film and the microstrip line are assumed to be infinitely long in the $z$-direction. Main panel: The solid line-the modulus of the amplitude of the in-plane microwave magnetic field $h_{\mathrm{x}}$ inside the film and behind it for the configurations (i) and (ii). The dashed line-the modulus of the amplitude of $h_{\mathrm{x}}$ for the configurations (iii) and (iv). These data are for the $x$-coordinate corresponding to the symmetry axis of the microstrip line. The coordinate $y=0(y=100 \mathrm{~nm})$ corresponds to the surface of the film, which faces (faces away from) the microstrip line. The microwave frequency is $15 \mathrm{GHz}$. 
used in the fields magnonics and spintronics. However, we assume that the metallic film is non-magnetic. We also note that the corresponding skin depth of a non-magnetic material with $\sigma=4.5 \times 10^{6} \mathrm{~S} / \mathrm{m}$ would be $\sim 1.94 \mu \mathrm{m}$ at $15 \mathrm{GHz}$ and that the results obtained below will be equally applicable to coplanar waveguides, which are also largely used as BSFMR transducers.

To find the field distributions created by the microwave current in the microstrip line, we employ an FDTD method described in Ref. 24. (However, we do not take into account the dynamic magnetisation, as the metal is non-magnetic.) In contrast to an analytical Fourier method also used in this work (see below), the FDTD method ${ }^{30}$ solves the full system of Maxwell's equations in the real time and space domains. An advantage of this approach is that we can take into account the realistic width of the microstrip line by approximating the line as an infinitesimally thin current sheet. ${ }^{24}$ Most significantly, the use of the current sheet approximation reduces the computation time as compared with the scenario when the thickness of the microstrip line is finite and also several orders of magnitude larger than the thickness of the conductive film.

The computation time reduction becomes very important when the FDTD method is applied to objects with one dimension significantly smaller than the others (see Refs. 24 and 31 and references therein). It is known that computational times on the order of a few periods of the source field are required to obtain a converged result. For example, for the microwave frequency $f=15 \mathrm{GHz}$ and the spatial discretisation $\Delta=1 \mathrm{~nm}$ across the film thickness, for one period of the source field the number of time steps required for the simulation is $\sim 21 \times 10^{6}$.

Another problem caused by the mismatch between the wavelength and the thickness of the metallic film is the inefficiency of the so-called absorbing boundary conditions (ABCs) such as the Mur's ABCs and Perfectly Matched Layers (PMLs) (see, e.g., Ref. 30 for more details). This problem is present in simulations conducted with FDTD software employing different versions of PMLs (see Ref. 30) and it arises because the computational domain is much smaller than the wavelength of the microwave wave.

In Ref. 24, we demonstrated that in the case of the perfect shielding this problem can be solved by using Perfect Electric Conductor (PEC) walls as the computational domain boundaries and moving them further from the far surface of the film (with respect to the microstrip line). The same approach is in general valid when the shielding is imperfect and the film becomes transparent for microwave radiation. However, one needs to keep in mind that in this case the unshielded wave can be reflected by the PEC wall back towards the far-film surface. Consequently, we extend the space behind the film as much as possible using all computational resources available to us. We also stop simulations before the reflected wave reaches the film. Previously, this approach was validated in our Ref. 25 by the comparison of results obtained using our FDTD method and our semi-analytical Bubnov-Galerkin method.

The main panel of Fig. 1 shows the modulus of the amplitude of the in-plane microwave magnetic field $h_{\mathrm{x}}$ across the film thickness $L=100 \mathrm{~nm}$ and in the free space region behind the film. This result was obtained by using the FDTD method. The microwave frequency is $15 \mathrm{GHz}$. Four representative film-microstrip configurations are considered: (i) the width $w$ of the microstrip line is infinite, (ii) $w=300 \mu \mathrm{m}$, (iii) $w=30 \mu \mathrm{m}$, and (iv) both the film and microstrip line have a finite width $w=30 \mu \mathrm{m}$. Whereas the scenario of $w \rightarrow \infty$ serves as a good approximation for a $1.5 \mathrm{~mm}$-wide microstrip line, ${ }^{8}$ which is prohibitively difficult to model using an FDTD method due to a ten orders of magnitude difference with the thickness $L$ of the conductive film, the values of $w$ in the remaining cases are chosen to be the same as in recent experimental works (see, e.g., Refs. 26 and 27).

One sees that for $w \rightarrow \infty$ [the configuration (i) in the inset in Fig. 1], the numerical simulations reproduce a wellknown result: ${ }^{6,24}$ the amplitude of $h_{\mathrm{x}}$ inside the film decays almost linearly from its maximum at the film surface facing the microstrip line to zero at the other film surface (the solid line in the main panel of Fig. 1). It is noteworthy that the same result holds for magnetic metallic films. In our Refs. 6 , 8,24 , and 25 , we use three different semi-analytical and numerical methods to rigorously solve the full system of the Maxwell's equations consistently with the linearised Landau-Lifshitz-Gilbert equation taking into account the exchange interaction. We demonstrate that the microwave magnetic field in sub-skin-depth magnetic metallic films is very similar to that in non-magnetic films of the same thickness and conductivity.

Most significantly, the result for $w \rightarrow \infty$ coincides to graphical accuracy with that for the configuration (ii) with $w=300 \mu \mathrm{m}$. (For the configuration (ii), the plotted field values are taken at the centre of the microstrip line.) This implies that the conductive film still acts as a perfect shield for $h_{\mathrm{x}}$ produced by a finite-width microstrip line. This result is in agreement with theoretical predictions for a coplanar line with a $350 \mu$ m-wide central conductor loaded by a conductive film of sub-skin-depth thickness. ${ }^{7}$

However, the profile of $h_{\mathrm{x}}$ changes dramatically for the configuration (iii), where $w=30 \mu \mathrm{m}$ [the dashed line in the main panel of Fig. 1]. One sees that the film is no longer a perfect shield for $h_{\mathrm{x}}$. The field decay is no longer linear and, most significantly, its profile is much more uniform. As shown in Ref. 8, the more pronounced uniformity of $h_{\mathrm{x}}$ has important implications for the excitation of SSWMs in highly conductive FM films.

The fact that $h_{\mathrm{x}}$ created by a $30 \mu \mathrm{m}$-wide stripline does not feel the presence of the film is convincingly confirmed by simulations for the configuration (iv) where the width of the film equals to the width of the microstrip line. Basically, in this case it is possible that the field penetrates into the area behind the film by rounding the sample. However, the corresponding profiles of $h_{\mathrm{x}}$ coincide to graphical accuracy with the profiles for the case (iii) where the film is continuous. This implies that the field passes through the film in this case as well.

Let us now try to explain the results above by using an analytical approach, which produces solutions in the Fourier space. This allows one to relate the presence or absence of the shielding effect to the width of the spectrum of spatial Fourier amplitudes of the microwave current density in the 
microstrip, and hence to the microstrip width. In our analysis, we focus on the region at the far surface of the conductive film (which is the surface which faces away from the microstrip line). It is known that for finite values of $w$ the in-plane $\left(h_{\mathrm{x}}\right)$ and out-of-plane $\left(h_{\mathrm{y}}\right)$ magnetic field components inside the film are related to each other. ${ }^{8,25}$ Let us analyse the quasi-static Maxwell's equations $\nabla \times \mathbf{h}=\sigma \mathbf{e}$, $\nabla \times \mathbf{e}=-i \omega \mu_{0} \mathbf{h}$, and also $\nabla \cdot \mathbf{h}=0$ in order to study this relationship. As we consider a non-magnetic metallic film, in these equations we omit the dynamic magnetisation term $\mathbf{m}$ and the electric bias currents. For the geometries in Figs. 1(ii) and 1(iii), one obtains

$$
\begin{gathered}
i k h_{y k}+\frac{\partial h_{x k}}{\partial y}=-\sigma e_{z k}, \\
\frac{\partial e_{z k}}{\partial y}=-i \omega \mu_{0} h_{x k}, \\
-i k h_{x k}+\frac{\partial h_{y k}}{\partial y}=0,
\end{gathered}
$$

where $\omega /(2 \pi)$ is the driving microwave frequency, $\sigma$ is the conductivity of the film, and all components of the microwave fields $\mathbf{h}$ and $\mathbf{e}$ are presented as Fourier expansions in the in-plane direction $x$ as

$$
\mathbf{h}, \mathbf{e}=\int_{-\infty}^{\infty} \mathbf{h}_{k}, \mathbf{e}_{k} \exp (i \omega t) \exp (-i k x) d x
$$

where $k$ is Fourier wave vector, which replaces the $x$-coordinate in Eqs. (1)-(3). For a magnetic film, $k$ would play the role of an in-plane wave number for a travelling spin wave. But in the present case of a non-magnetic film, $k$ does not have a particular physical meaning. However, the introduction of $k$ results in simple analytical formulas, which allow one to relate the main features of the $y$-profile of the microwave magnetic field to some characteristic scale of the inplane non-uniformity of the microwave current density. The wave vector $k$ is not directly present in our FDTD simulations (Fig. 1) but, as we will show below, a characteristic value of $k_{\max }$ can be assigned to any particular geometry. Essentially, the narrower the microstrip, the larger $k_{\max }$ is.

By differentiating Eq. (1) and combining it with Eqs. (2) and (3), one obtains

$$
\begin{aligned}
& \frac{\partial^{2} h_{x k}}{\partial y^{2}}-K^{2} h_{x k}=0, \\
& h_{y k}-\frac{i k}{K^{2}} \frac{\partial h_{x k}}{\partial y}=0,
\end{aligned}
$$

where $K^{2}=i \sigma \omega \mu_{0}+k^{2}$ inside the film. Outside the film $\sigma=0$ and $K^{2}$ reduces to $k^{2}$. (To derive Eq. (6), we also used the relation $k e_{z k}=-\omega \mu_{0} h_{y k}$ obtained from the Maxwell's equations.) The fields outside the film are known to constants only. We require that the magnetic field vanishes at $y= \pm \infty$. Thus, outside the film (where $\sigma=0) h_{x k}=\exp ( \pm|k| y) C$ and from Eq. (6) $h_{y k}=-i C|k| \exp ( \pm|k| y) / k$, being $C$ an arbitrary constant, and the negative (positive) sign is for the area behind (in front of) the film. Then, from the conditions of continuity of $h_{\mathrm{x}}$ and $e_{\mathrm{z}}$ at the surfaces of the film we obtain the following conditions at the surfaces of the film from inside the film:

$$
\begin{aligned}
& h_{x k}+i h_{y k} \frac{k}{|k|}=0, \quad \text { for } \quad y=L \\
& h_{y k}-i h_{x k} \frac{k}{|k|}=j_{k} \frac{k}{|k|}, \quad \text { for } \quad y=0,
\end{aligned}
$$

where $j_{k}$ is the linear current density for the microwave current sheet. (The sheet located at $y=0$.) The analytical solution of Eqs. (5) and (6) with boundary conditions Eq. (7) is very simple

$$
\begin{aligned}
& h_{x k}=K \frac{K \sinh [K(y-L)]-k \cosh [K(y-L)]}{\left(K^{2}+k^{2}\right) \sinh (K L)+2 k K \cosh (K L)} j_{k} \\
& h_{y k}=-i|k| \frac{k \sinh [K(y-L)]-K \cosh [K(y-L)]}{\left(K^{2}+k^{2}\right) \sinh (K L)+2 k K \cosh (K L)} j_{k},
\end{aligned}
$$

where $K$ is the principal square root of the complex value $i \sigma \omega \mu_{0}+k^{2}$.

The expression for $K$ above can be cast in the following form: $K^{2}=k^{2}\left(1+2 i /(k \delta)^{2}\right)$, where $\delta=1 / \sqrt{2 \sigma \omega \mu_{0}}$ is the microwave skin depth. From this expression and Eq. (5), one sees that the effect of the conductivity should become negligible for $k \delta \gg 1$. Figure 2 shows the field profiles across the film thickness for three different values of $k: k=0$, $k_{1}=730 \mathrm{~cm}^{-1}$, and $k_{2}=7300 \mathrm{~cm}^{-1} \cdot 32$ The second and third $k$-values have been chosen such that $k_{1}=0.1\left(\sigma \omega \mu_{0}\right)^{1 / 2}$ $=0.1 /(\sqrt{2} \delta)$ and $k_{2}=1 /(\sqrt{2} \delta)$, respectively. We note that the value $k_{2}$ is special because numerical instability arises when one tries to solve Eqs. (1)-(3) in the presence of the dynamic magnetisation $\mathbf{m}$ by using a finite difference method. $^{25}$

One sees that for $k=0$ the $h_{x k}$-component [solid line in Fig. 2(a)] is perfectly shielded and the $h_{y k}$-component (dotted line) is zero everywhere, including $y=0$ and $y=L$, which is required to fulfil the conditions given by Eq. (7). ${ }^{33}$ For a larger $k$-value $k_{1}=730 \mathrm{~cm}^{-1}$, the metal film becomes transparent for the microwave magnetic field. One sees that in this case $h_{y k}$ [dashed-dotted line in Fig. 2(a)] has almost the same amplitude as $h_{x k}$. However, $h_{y k}$ is not shielded (i.e., it is independent of the $y$-coordinate), whereas some shielding of the $h_{x k}$-component (dashed line) is still remaining. Note that in order to fulfil the conditions given by Eq. (7) at $y=L$, it should stand $\left|h_{\mathrm{xk}}\right|=\left|h_{\mathrm{yk}}\right|$. Furthermore, for a significantly larger $k_{2}=7300 \mathrm{~cm}^{-1}$ [Fig. 2(b)] the relationship $\left|h_{x k}\right|=\left|h_{y k}\right|$ holds across the whole film thickness. As follows from Fig. 3(a), the relationship $\left|h_{x k}\right|=\left|h_{y k}\right|$ roughly holds for $k>3000 \mathrm{~cm}^{-1}$ (or $k \delta>0.3$ ).

More clearly, this effect is seen in Fig. 3 where we demonstrate the values of the two field components at the film surfaces as a function of $k$. From this figure, one sees that at the far film surface $(y=L)$ it always holds that $\left|h_{y k}\right|=\left|h_{x k}\right|$, and at the front film surface $(y=0) h_{x k}$ drops with $k$, but $h_{y \mathrm{k}}$ shows the opposite tendency.

More insight into the physics may be gained by looking at the ratio of the field amplitudes at an arbitrary point $y$ to the respective values at $y=0$. Figure 4 demonstrates this 

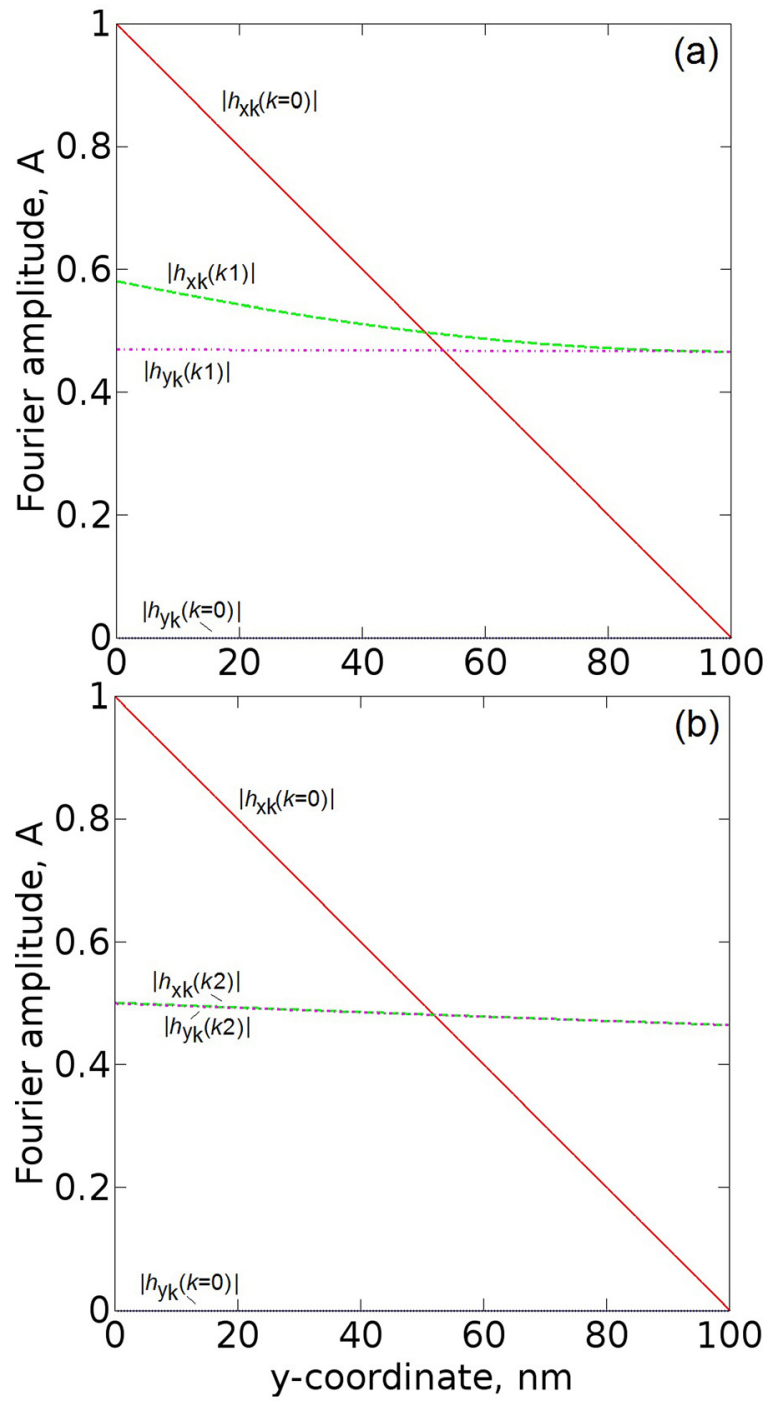

FIG. 2. Profiles of the $h_{x k^{-}}$and $h_{\mathrm{yk}}$-components of the microwave magnetic field as a function of the $y$-coordinate across the film thickness. The solid and dotted lines denote, respectively, $\left|h_{x k}\right|$ and $\left|h_{y k}\right|$ for $k=0$. The values of $\left|h_{x k}\right|$ and $\left|h_{y k}\right|$ for a non-zero $k$-value are denoted by the dashed and dasheddotted line, respectively. The non-zero $k$-value is $k_{1}=730 \mathrm{~cm}^{-1}$ in (a) and $k_{2}=7300 \mathrm{~cm}^{-1}$ in (b). All profiles were calculated for the microwave frequency $15 \mathrm{GHz}$ by using Eq. (8). In these calculations, it is assumed that $j_{\mathrm{k}}=1 \mathrm{~A}$ for any $k$-value.

result. From this figure, one sees that the out-of-plane component $h_{y k}$ is not shielded at all. Also, from the comparison of Figs. 3 and 4 one finds that the film becomes transparent for the microwave radiation when $h_{y k}$ becomes significant. Therefore, it is reasonable to interpret the transparency of the film as mediated by the out-of-plane component $h_{y k}$. This component is not shielded, easily passes the film and induces the in-plane component $\left(h_{x k}\right)$ of the field because the field lines should be closed.

The results presented in Fig. 3 confirm the theoretical predictions from Ref. 8 that the shielding is perfect $\left[h_{x k}(y=L)=0\right.$ ] for $k=0$, but it rapidly reduces with an increase in $k$. Most significantly, the width of the microstrip line $w$ is indirectly present in Eqs. (6)-(8). Indeed, for a sheet of width $w$ (along the $x$-direction in Fig. 1) of a uniform current flowing in the direction $z$, the Fourier image of the current density is $j_{k}=(w /(2 \pi)) \sin (k w / 2) /(k w / 2)$. This implies
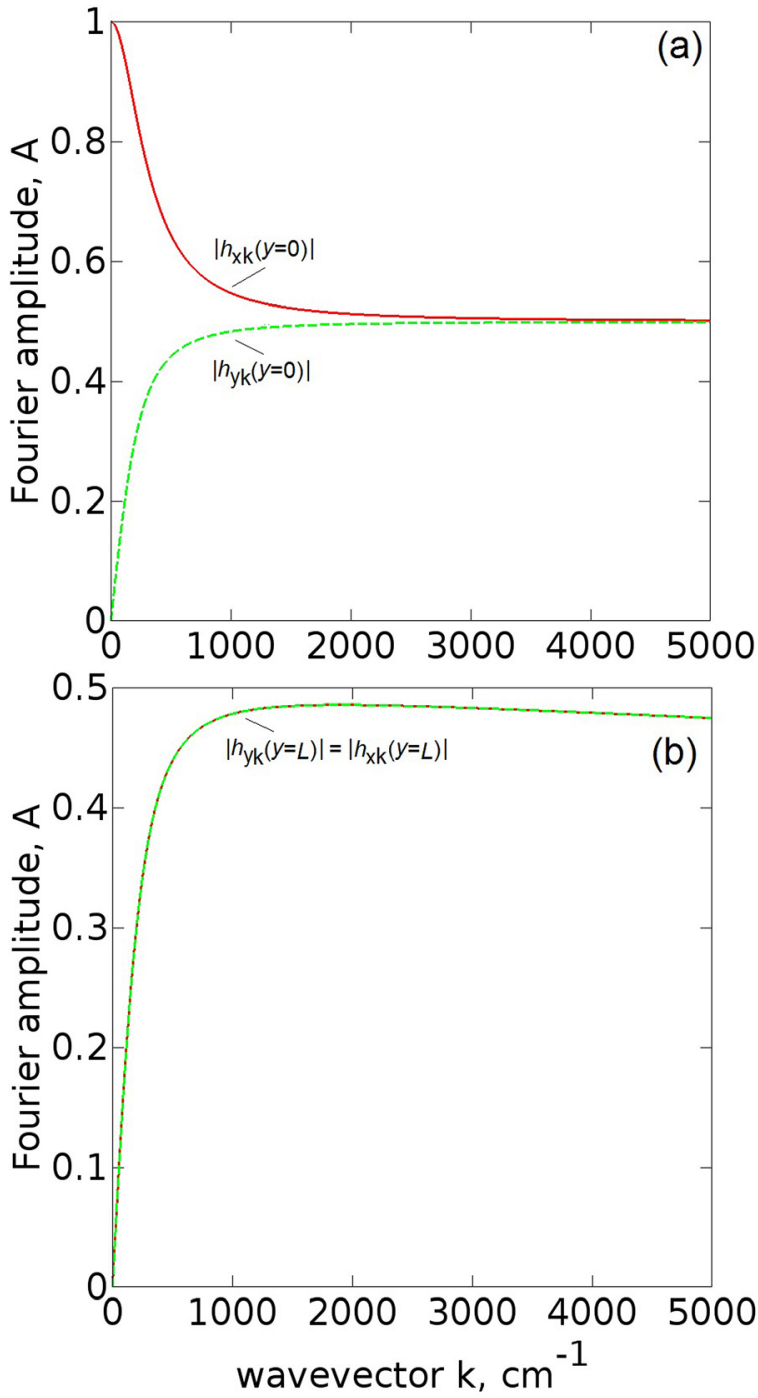

FIG. 3. Profiles of the $h_{x k^{-}}$and $h_{\mathrm{yk}}$-components of the microwave magnetic field as a function of the wave vector $k$ at the surfaces of the film: (a) $y=0$ (the surface of the film which faces the microstrip line) and (b) $y=L$ (the surface of the film which faces away from the microstrip line). Note that in (b) $\left|h_{y k}\right|=\left|h_{x k}\right|$ for all $k$-values. The microwave frequency is $15 \mathrm{GHz}$. In these calculations, it is assumed that $j_{\mathrm{k}}=1 \mathrm{~A}$ for any $k$-value.

that the Fourier spectra of $h_{x k}$ and $h_{y k}$ are concentrated between $-k_{\max }$ and $+k_{\max }$, where $k_{\max }=2 \pi / w$ (see, e.g., Ref. 8 for microstrip lines and Ref. 34 for coplanar lines). In the limit $w \rightarrow \infty$, one obtains $k_{\max }=0$. This implies that for a sheet of microwave current of an infinite width $h_{x}$ is perfectly shielded. For a finite $w$, a range of unshielded Fourier components appears.

Figure 5 demonstrates the calculated spectra of $h_{x k}$ and $h_{y k}$ at $y=L$ and $y=0$ for two different values of $w$ and for $j_{k}$ given by the formula above. Both spectra for $y=L$ [Figs. 5(a) and 5(b)] are characterised by significantly smaller values of $h_{x k}$ for $k<100 \mathrm{~cm}^{-1}$ with respect to $y=0$ [Figs. 5(c) and 5(d), respectively]. This is due to strong shielding of these Fourier components of the field by the metallic film [see the respective $k$-area in Fig. 3(b)]. For $w=300 \mu \mathrm{m}$, the first zero of $j_{k}(k)$ (and hence of $h_{x k}(k, y)$ ) is located at $k_{\max }= \pm 209 \mathrm{~cm}^{-1}$. One sees that for $y=0$ most of the spectral density is located between $-k_{\max }$ and $+k_{\max }$. However, 


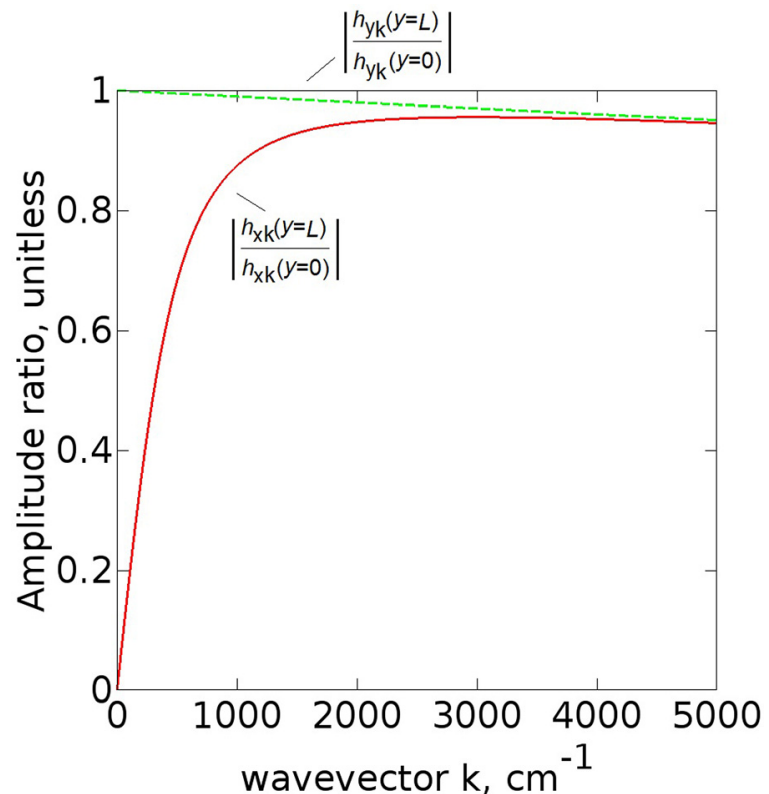

FIG. 4. Amplitude ratio $\left|h_{x k}(y=L)\right||| h_{x k}(y=0) \mid$ (solid line) and $\left|h_{y k}(y=L)\right| \mid$ $\left|h_{y k}(y=0)\right|$ (dashed line) as a function of the wave vector $k$. The microwave frequency is $15 \mathrm{GHz}$.

for $y=L$ the Fourier-amplitudes in this area are smaller than for $y=0$ by at least ten times. This is in agreement with Fig. 4, which demonstrates that for $k_{\max }$ the value of $\left|h_{x k}(k, y=L) /\right| h_{x k}(k, y=0) \mid$ reaches just $\sim 50 \%$. Accordingly, the amplitude of $h_{x}(y=L)$ in Fig. 1 (solid line) is small with respect to $y=0$, since the $x$-profile of the field from Fig. 1 is essentially given by inverse Fourier transformation of Fig. 5(b). For $w=30 \mu \mathrm{m}$, one obtains $k_{\max }=2090 \mathrm{~cm}^{-1}$, Thus, the spectrum of $j_{\mathrm{k}}(k)$ extends well into the range of unshielded Fourier components of $h_{x k}(k, y=L)$ from Fig. 4. (We may define the spectrum of unshielded components as one for which $\left|h_{x k}(k, y=L) /\right| h_{x k}(k, y=0) \mid>0.8$ or so. In Fig. 4, this range corresponds to $k>500 \mathrm{~cm}^{-1}$.) From the comparison of Figs. 5(a) and 5(c), one sees that the spectrum of the shielded components is quite narrow and the total spectral density contained in this range is insignificant with respect to the whole spectrum. This explains well why the $h_{x}$-field in Fig. 1 (dashed line) is shielded very weakly.

Furthermore, from this figure one also sees that the $h_{x k}$ spectrum is an even function of $k$, but the $h_{y k}$ spectrum is an odd function. This implies that $h_{x k}$ is an even function of $x$ and $h_{y}$ is an odd function of $x$ with a node at $x=0$. Since $h_{y}(x)$ is a spectrally limited function, based on this anti-symmetric spectrum one may expect two maxima of $\left|h_{y}(x)\right|$ at some distance from each other. This conclusion is in agreement with the numerical FDTD results presented Fig. 6, where one clearly sees two extrema of $h_{y}(x)$ at the edges of the current sheet.

To summarise this section, we conclude that spatially extended sources of the field are well shielded by sub-skindepth metallic layers because $k_{\max }$ for them is small and thus the $h_{y}$ component for them is almost vanishing. On the contrary, highly localised sources located close to the metallic shields are characterised by much larger $k_{\max }$-values for the
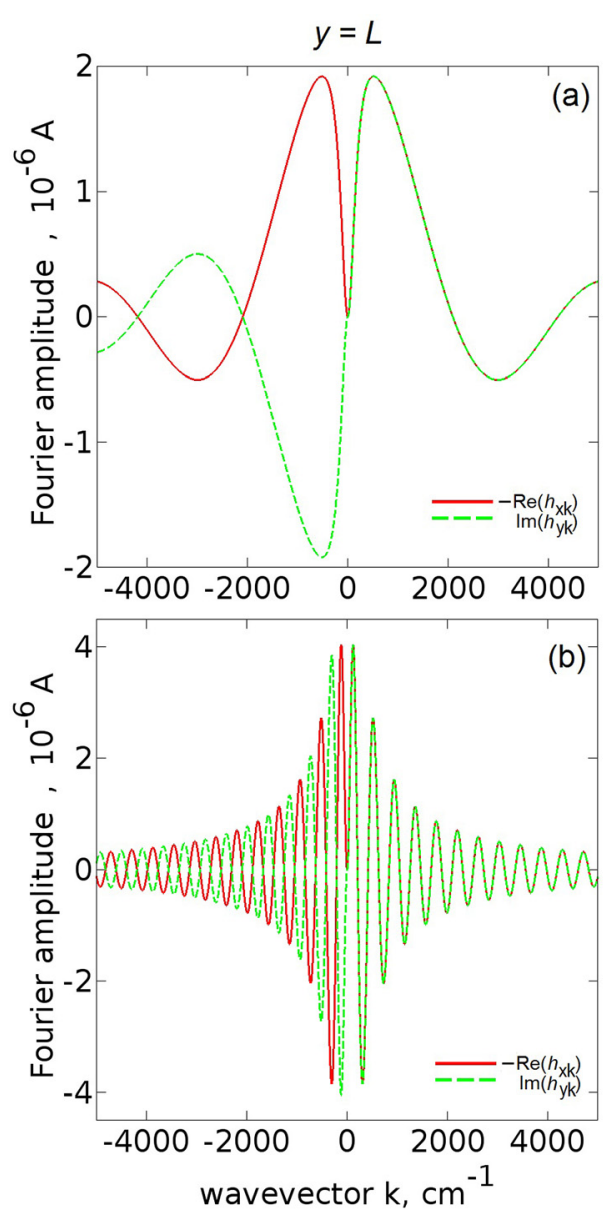

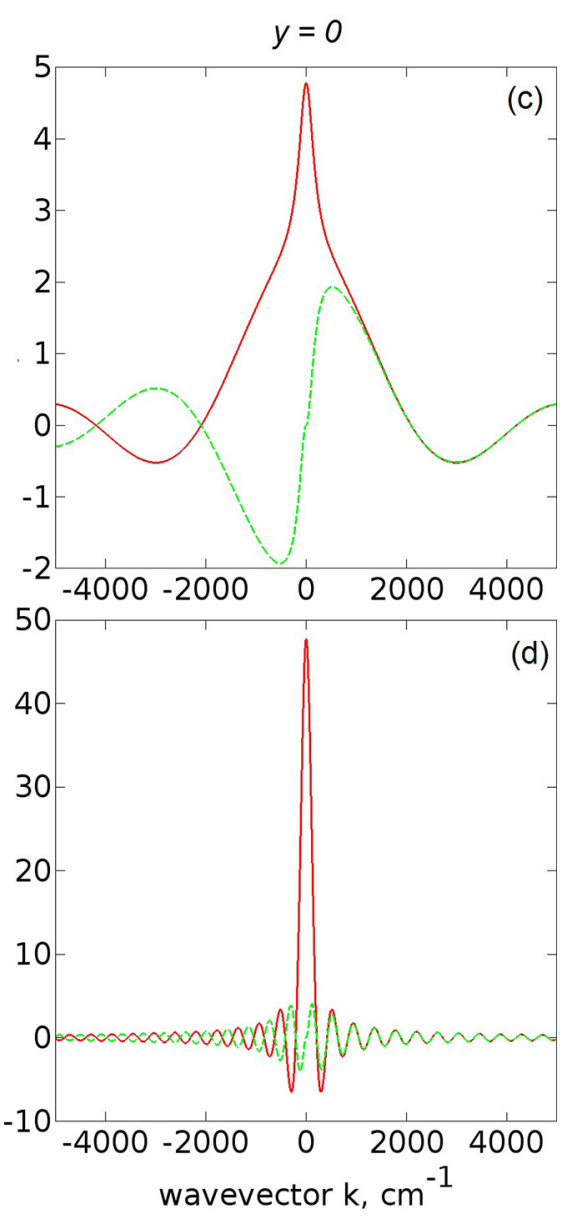

FIG. 5. Profiles of $-\operatorname{Re}\left(h_{x k}\right)$ and $\operatorname{Im}\left(h_{\mathrm{yk}}\right)$ for $w=30 \mu \mathrm{m}$ ((a) and (c)) and $w=300 \mu \mathrm{m}((\mathrm{b})$ and (d)) calculated by taking into account the realistic dependence of $j_{\mathrm{k}}$ on the wave vector $k$. In panels ((a) and (b)), the $y$-coordinate corresponds to the surface of the film that faces away from the microstrip line (i.e., $y=L$ ). In panels ((c) and (d)), the $y$-coordinate corresponds to the surface of the film that faces the microstrip line (i.e., $y=0$ ). The microwave frequency is $15 \mathrm{GHz}$. It is assumed that the current density $j$ in the microstrip is $1 \mathrm{~A} / \mathrm{m}$ (not to confuse with the Fourier image of the current density $j_{k}$ from the captions to Figs. 2 and 3). 

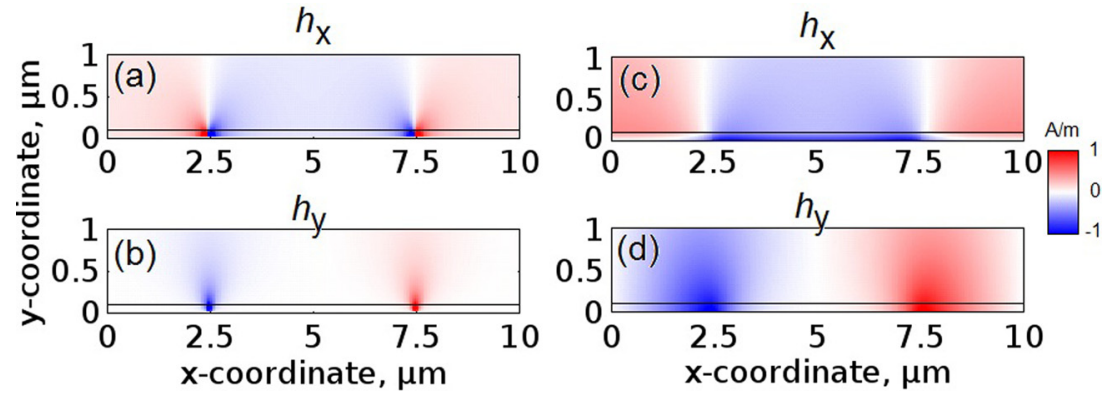

FIG. 6. False colour profiles of the amplitude of (a) in-plane microwave magnetic field $h_{x}$ and (b) the out-of-plane microwave magnetic field $h_{y}$ in the continuous $100 \mathrm{~nm}$ thick metal film and the $900 \mathrm{~nm}$-thick free space region behind it. The surface of the film facing away from the microstrip line is denoted by the straight horizontal lines. For the sake of illustration, the width of the microstrip line is chosen to be $5 \mu \mathrm{m}(2.5 \mu \mathrm{m}<x<7.5 \mu \mathrm{m})$. The microwave frequency is $15 \mathrm{GHz}$. (c) and (d) The same as in the panels (a) and (b) but with a $1 \mu \mathrm{m}$-thick microstrip line made of copper $\left(\sigma=6 \times 10^{7} \mathrm{~S} / \mathrm{m}\right)$. Note a good qualitative agreement between the results obtained using the current sheet model of the microstrip line, which justifies the use of this approximation. Also, note a larger contrast of false colour in the region inside the film and behind it in (c) as compared to (a), which evidences stronger shielding of $h_{x}$ in (c) as compared to (a).

out-of-plane component of the magnetic field. For this reason, $h_{y}(x)$ for them represents two narrow peaks. Due to its strong localisation, $h_{y}$ easily "pierces" the metallic shield and induces an in-plane $\left(h_{x}\right)$ field behind the shield. This inplane field closes the loops of the field by joining the two maxima of $\left|h_{y}(x)\right|$. A larger (mean) distance of the localised source from the shield surface, or equivalently a source more extended in the normal direction is shielded more significantly (Fig. 6). ${ }^{35}$ This conclusion is in agreement with the classical theory of shielding by Schelkunoff. ${ }^{36}$

\section{SHIELDING BY PERIODIC CONDUCTIVE NANOSTRUCTURES}

Periodic nanostructures consisting of ferromagnetic metal stripes separated by air slits_-one-dimensional magnonic crystals-have recently attracted considerable attention due to their ability to manipulate spin waves at will. ${ }^{4}$ Apart from a strong reduction of the amplitude of the BSFMR response, in combination with the effect of eddy currents nano-patterning adds interesting features to measured BSFMR absorption traces. For example, both odd (anti-symmetric) and even (symmetric)
SSWMs across the width of the ferromagnetic metallic nanostripes can be excited ${ }^{28}$ in contrast to magneto-insulating nanostructures where the excitation of only symmetric modes is supported. The explanation of this effect is possible by drawing analogy with continuous conductive films. For continuous metallic films due to the effect of eddy currents, the microwave Oersted field is anti-symmetric across the film thickness. The same interpretation might be applied to highly conductive nanostructures. However, there are some peculiarities associated with the nano-patterning, which we demonstrate below.

The inset in Fig. 7(a) schematically shows the geometry of the investigated nanostructure. To be consistent with the previous discussion, we choose the thickness of the highly conductive (metallic) nanostripes to be $L=100 \mathrm{~nm}$. The remaining parameters of the nanostructure are the constant stripe width $H=340 \mathrm{~nm}$ (Ref. 18) and the slit width $g$, which is varied. The microwave frequency is $15 \mathrm{GHz}$. Also, as in the experiment from Ref. 28 the width $w$ of the microstrip was $0.3 \mathrm{~mm}$, we model this microstrip as the one having $w \rightarrow \infty$.

We employ the same FDTD method as used in Sec. II to investigate the shielding by continuous metal films. One sees

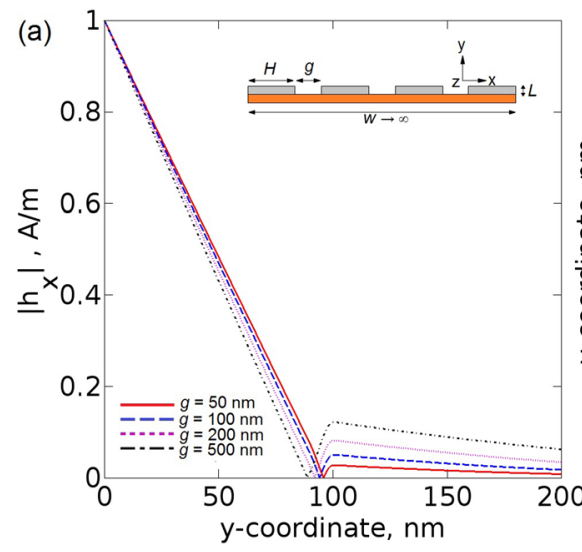

(b)

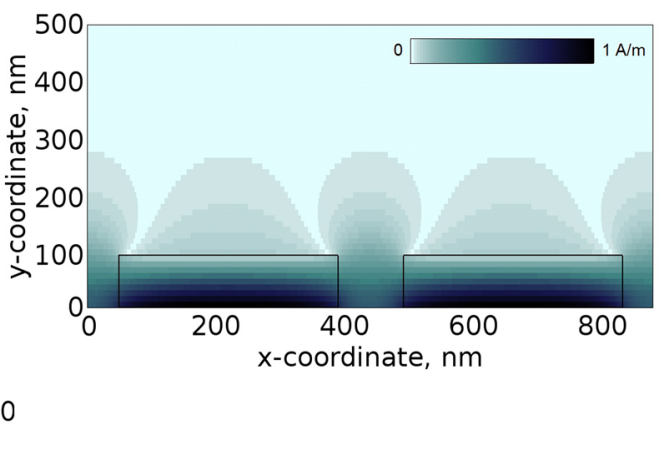

FIG. 7. (a) Inset: Schematic of the periodic nanostructure consisting of alternating nanostripes of the thickness $L=100 \mathrm{~nm}$. The nanostripes are infinite along the $z$-axis. The microstrip line is infinitely long in the $x$-direction. The microwave current in the microstrip line flows in the $z$-direction (towards the reader). Main panel: the modulus of the amplitude of the in-plane microwave magnetic field $h_{x}$ across the thickness of one of the conductive nanostripes and behind it at $x=210 \mathrm{~nm}$ (see panel (b)). Thick solid line: $g=50 \mathrm{~nm}$, dashed line: $g=100 \mathrm{~nm}$, dotted line: $g=200 \mathrm{~nm}$, and the dashed-dotted line: $g=500 \mathrm{~nm}$. The coordinate $y=0(y=100 \mathrm{~nm})$ corresponds to the surface of the Permalloy nanostructure which faces (faces away from) the microstrip line. (b) False colour profile of $\left|h_{x}\right|$. Two periods of the nanostructure are shown. The contours of the nanostripes are denoted by the straight lines. In all panels, the microwave frequency is $15 \mathrm{GHz}$. 
[the main panel of Fig. 7(a)] that microwave magnetic field is not perfectly shielded by the metallic nanostructure. In fact, the field leaks through the air slits between the nanostripes [Fig. 7(b)] and forms an evanescent field. This near field is localised in the closest vicinity of the nanostripes. Overall, the $h_{x}$-profile resembles the one in Fig. 1 (solid line) with the only difference that $h_{x}$ does not completely vanish at the far surface of the nanostructure.

The physical mechanism of the field leakage may be explained similarly to the above considered case of a narrow microstrip line. Similarly to the strong concentration of the $h_{y}$-component at the edges of the current sheet, there is a strong concentration of the $h_{y}$-component of the eddy current induced in the stripes at the stripe edges, both inside the stripes and in the gaps. ${ }^{28}$ As we have seen above, metallic shields are transparent to $h_{y}$ of highly localised sources. The eddy current at nanostripe edges acts as such a localised source of $h_{y}$, even when the external microwave field source does not possess an $h_{y}$ field component (as in the case of a very wide microstrip line). Accordingly, the $h_{y}$-field leaks through the gaps between the nanostripes and through the metal near the gap edges and forms closed loops behind the material by transforming itself into an in-plane field $\left(h_{x}\right)$ at some distance $x$ from the gap edge.

\section{DISCUSSION}

In this section, we discuss the implications of the eddycurrent shielding for BSFMR measurements. We also discuss the applicability of our findings to the research on magnetically tuneable metamaterials where the eddy-current shielding effect has been known but it is often regarded as potentially parasitic.

To start with, we show that the width of the gap between the metallic nanostripes of the periodic structure in the inset in Fig. 7(a) affects the strength of the shielding effect. Hereafter, we assume that the width of the nanostripes $H$ is constant. In Fig. 7(a), one sees that for a small gap width $(g=50 \mathrm{~nm})$ the $h_{x}$-component distribution resembles the one in the continuous film (Fig. 1, solid line). However, the amplitude of the $h_{x}$-component behind the film increases as $g$ is increased. Due to computation constraints, in our simulations the maximum attainable gap width was $g=500 \mathrm{~nm}$. For this value, the leakage through the slits is maximum, which results in a modified $h_{x}$-component profile [the dashed-dotted curve in Fig. 7(a)]. First, in the region $0<y<90 \mathrm{~nm}$ (inside the conductive nanostripe) this curve has the maximum slope as compared with the curves for $g=50,100$, and $200 \mathrm{~nm}$. Second, the curve changes its sign inside the nanostripe in the region close to the metal-air interface at $y=100 \mathrm{~nm}$. The change in sigh may appear because the origins of the $h_{x}$-component inside and behind the film are different.

Indeed, the field inside the metallic nanostripe is a combination of the Oersted field of the microwave current in the microstrip and the Oersted field of the eddy currents in the nanostripe. The field of the microstrip is uniform along $y$. For a continuous film, the field of the Oersted current is perfectly anti-symmetric across the film thickness. The two fields perfectly cancel each other at $y=L$, but for $y<L$ the larger contribution always originates from the microstrip field. This results in $h_{x}$ of the same sign all across the film thickness. For the nanostripe array, the Oersted field of the eddy currents is modified and seems to over-compensate the microstrip field near $y=0$, as the profiles in Fig. 7(a) suggest.

How might this behaviour of the $h_{x}$-field inside the conductive stripes affect the excitation of the fundamental and higher-order SSWMs in realistic one-dimensional Permalloy magnonic crystals? First, in agreement with the previous theories for continuous ferromagnetic metal films $\mathrm{s}^{6,24}$ and a relevant experiment, ${ }^{8}$ one may expect an increase in the amplitude of the SSWMs due to a more pronounced inhomogeneity of the $\left|h_{\mathrm{x}}\right|$-profile. Indeed, spin waves in periodic nanostructures consisting of very wide ferromagnetic metal nanostripes, which are the case of this work, are expected to obey the dispersion law for spin waves in continuous ferromagnetic metal films. ${ }^{18}$ Second, a recent experiment ${ }^{28}$ demonstrated that the higher-order absorption peaks corresponding to anti-symmetric standing-wave resonances along $x$ appear in the FMR spectrum of periodic ferromagnetic metal nanostructures due to the contribution from eddy currents. Thus, any alteration of the eddy-current effect, including one due to an increase in the gap size between the nanostripes, may affect the excitation of these modes.

Our results can also be applied to research on magnetically tuneable metamaterials whose resonances are also exploited in a BSFMR arrangement (see, e.g., Refs. 20 and 21). Currently, as shown in the cited papers, the use of continuous ferromagnetic metal films in magnetically tuneable metamaterials is avoided due to the perfect eddy-current shielding of the metamaterial by such films. In order to circumvent this effect, one often uses either magneto-insulating YIG films ${ }^{20}$ or micro-patterned ferromagnetic metal structures. ${ }^{21}$ However, high-quality YIG films are relatively expensive and are not compatible with the CMOS platform. Furthermore, micro-patterning complicates the structure of the metamaterial and understanding of its operation. We argue that thin ferromagnetic metallic films (thickness $<100 \mathrm{~nm}$ ), which are compatible with the CMOS platform, can be used in metamaterials employing, for example, large arrays of split-ring resonators. This is achievable by reducing the width of the metallic strips that form the splitring resonators from the currently used $\sim 300 \mu \mathrm{m}$ (Refs. 20 and 21) to $\sim 30 \mu \mathrm{m}$ or oven smaller. As shown in Fig. 1 (dashed line), the microwave magnetic field created by strips with such dimensions is virtually not shielded by continuous conductive thin films. This eliminates the need in film micro-patterning. Getting rid of the micropatterning eliminates the drawback of a strong dependence of the metamaterial frequency on the patterned element size. ${ }^{21}$

\section{CONCLUSIONS}

We have theoretically demonstrated that in broadband stripline ferromagnetic resonance measurements of magnetisation dynamics in highly conductive continuous thin films the eddy-current shielding is perfect only when the width of the microstrip line is essentially macroscopic $(>300 \mu \mathrm{m}$ in our example), which agrees with the previous theoretical 
results in Refs. 7 and 25. The shielding effect gradually disappears when the width of the microstrip line is decreased. For the standard conductivity of permalloy $\left(\mathrm{Ni}_{80} \mathrm{Fe}_{20}\right)$, the shielding effect almost vanishes when the width of the microstrip line is $\leq 30 \mu \mathrm{m}$. This is due to strong concentration of the out-of-plane component of the microwave magnetic field induced by the stripline at the edges of the strip. A highly concentrated (localised) field is not shielded by the film of a sub-skin-depth thickness. The localised out-ofplane field penetrates through the film and gradually converts itself into the in-plane magnetic field in the area behind the film. This produces an effect similar to extraordinary transparency known from nano-optics. ${ }^{37}$ However, the physical interpretation of the present effect is different: whereas the excitation of a surface wave is the prerequisite for extraordinary transparency in nano-optics, in our case the prerequisite is the excitation of eddy currents. Since our films and nanostructures are of sub-skin-depth thicknesses and the nanostructures are also of the sub-skin-depth sizes in the sample plane, the eddy currents flow through the whole bulk of the material with (nearly) uniform current density across the volume of the material.

Based on these results, we have also given recommendations for the development of magnetically tuneable metamaterials. We argue that a decrease in the width of the metallic strips of split-ring resonators (or meta-atoms of other shapes such as, e.g., crosses) will minimise the adverse effect of eddy currents on the performance of metamaterials. Finally, we note that our findings might be useful for the design of high-frequency integrated circuits where the problem of the passive element shielding still remains unsolved. ${ }^{1}$

\section{ACKNOWLEDGMENTS}

This work was supported by the UWA UPRF scheme and the Australian Research Council.

${ }^{1}$ T. S. D. Cheung and J. R. Long, IEEE J. Solid-State Circuits 41, 1183 (2006).

${ }^{2}$ B. E. Herr, J. Blacke, and R. D. Paynton, Inf. Disp. 5, 30 (2010).

${ }^{3} \mathrm{H}$. Ott, Electromagnetic Compatibility Engineering (John Wiley \& Sons, New York, 2009).

${ }^{4}$ V. V. Kruglyak, S. O. Demokritov, and D. Grundler, J. Phys. D.: Appl. Phys. 43, 264001 (2010).

${ }^{5}$ R. L. Stamps, S. Breitkreutz, J. Åkerman, A. V. Chumak, Y.-C. Otani, G. E. W. Bauer, J.-U. Thiele, M. Bowen, S. A. Majetich, M. Kläui, I. L. Prejbeanu, B. Dieny, N. M. Dempsey, and B. Hillebrands, J. Phys. D: Appl. Phys. 47, 333001 (2014).

${ }^{6}$ M. Kostylev, J. Appl. Phys. 106, 043903 (2009).

${ }^{7}$ M. Bailleul, Appl. Phys. Lett. 103, 192405 (2013).

${ }^{8}$ K. J. Kennewell, M. Kostylev, N. Ross, R. Magaraggia, R. L. Stamps, M. Ali, A. A. Stashkevich, D. Greig, and B. J. Hickey, J. Appl. Phys. 108, 073917 (2010).
${ }^{9}$ C. T. Boone, H. T. Nembach, J. M. Shaw, and T. J. Silva, J. Appl. Phys. 113, 153906 (2013).

${ }^{10}$ H. Głowiński, M. Schmidt, I. Gościańska, J.-Ph. Ansermet, and J. Dubowik, J. Appl. Phys. 116, 053901 (2014).

${ }^{11}$ A. Fessant, J. Geraltowski, J. Loaec, and H. Legall, J. Magn. Magn. Mater, 83, 557 (1990).

${ }^{12}$ T. J. Silva, C. S. Lee, T. M. Crawford, and C. T. Rogers, J. Appl. Phys. 85, 7849 (1999).

${ }^{13}$ G. Counil, J.-V. Kim, T. Devolder, C. Chappert, K. Shigeto, and Y. Otani, J. Appl. Phys. 95, 5646 (2004).

${ }^{14}$ M. L. Schneider, A. B. Kos, and T. J. Silva, Appl. Phys. Lett. 85, 254 (2004).

${ }^{15}$ S. S. Kalarickal, P. Krivosik, M. Wu, C. E. Patton, M. L. Schneider, P. Kabos, T. J. Silva, and J. P. Nibarger, J. Appl. Phys. 99, 093909 (2006).

${ }^{16}$ C. Bilzer, T. Devolder, P. Crozat, C. Chappert, S. Cardoso, and P. P. Freitas, J. Appl. Phys. 101, 074505 (2007).

${ }^{17}$ Y. V. Khivintsev, L. Reisman, J. Lovejoy, R. Adam, C. M. Schneider, R. E. Camley, and Z. J. Celinski, J. Appl. Phys. 108, 023907 (2010).

${ }^{18}$ C. S. Chang, M. Kostylev, A. O. Adeyeye, M. Bailleul, and S. Samarin, Europhys. Lett. 96, 57007 (2011).

${ }^{19}$ F. Falcone, F. Martín, J. Bonache, R. Marqués, and M. Sorolla, Microw. Opt. Technol. Lett. 40, 3 (2004).

${ }^{20}$ G. B. G. Stenning, G. J. Bowden, L. C. Maple, S. A. Gregory, A. Sposito, R. E. Eason, N. I. Zheludev, and P. A. J. de Groot, Opt. Express 21, 1456 (2013).

${ }^{21}$ S. A. Gregory, G. B. G. Stenning, G. J. Bowden, N. I. Zheludev, and P. A. J. de Groot, New. J. Phys. 16, 063002 (2014).

${ }^{22}$ R. A. Shelby, D. R. Smith, and S. Schultz, Science 292, 77 (2001).

${ }^{23}$ D. Schurig, J. J. Mock, B. J. Justice, S. A. Cummer, J. B. Pendry, A. F. Starr, and D. R. Smith, Science 314, 977 (2006).

${ }^{24}$ I. S. Maksymov and M. Kostylev, J. Appl. Phys. 113, 043927 (2013).

${ }^{25}$ I. S. Maksymov and M. Kostylev, J. Phys. D: Appl. Phys. 46, 495001 (2013).

${ }^{26}$ J. Ding and A. O. Adeyeye, Appl. Phys. Lett. 101, 103117 (2012).

${ }^{27}$ J. Ding, M. Kostylev, and A. O. Adeyeye, Phys. Rev. B 84, 054425 (2011).

${ }^{28}$ M. Kostylev, J. Ding, E. Ivanov, S. Samarin, and A. O. Adeyeye, J. Appl. Phys. 115, 173903 (2014).

${ }^{29}$ In a relevant experiment, ${ }^{28}$ the width of the microstrip line is $0.3 \mathrm{~mm}$. In agreement with our Refs. 6 and 24, microstrip lines of such a width can be considered as infinitely wide.

${ }^{30} \mathrm{~A}$. Taflove and S. C. Hagness, Computational Electrodynamics: The Finite-Difference Time-Domain Method, 3rd ed. (Artech House Publishers, Boston, 2005).

${ }^{31}$ H. Zhao, S. Crozier, and F. Liu, Appl. Math. Model. 27, 409 (2003).

${ }^{32}$ For simplicity, we assume that $k$ is positive. This eliminates $k$ from Eq. (7).

${ }^{33}$ Note that for the specific case $k=0$, in magnetic materials, although the eddy current-induced $h_{y k}$ is vanishing, there is one more component of $h_{y k}$ which does not vanish. It is of magnetostatic nature and is given by an equation $\operatorname{divh} \mathbf{h}_{k}=-\operatorname{divm}_{k}$, which is not a part of the models (1)-(3). For $k \neq 0$, the eddy-current and the magnetostatic contributions do not separate and are described by the models (1)-(3) with extra terms which depend on $\mathbf{m}_{k}$.

${ }^{34}$ K. J. Kennewell, M. Kostylev, and R. L. Stamps, J. Appl. Phys. 101, 09D107 (2007).

${ }^{35}$ The retardation is not included in our models (1)-(3) and (6)-(8), so we are considering only sources of a near field, such as a stripline.

${ }^{36}$ S. A. Schelkunoff, Electromagnetic Waves (D. Van Nostrand Company, New York, 1948), pp. 303-315.

${ }^{37}$ F. J. Garcia-Vidal, L. Martin-Moreno, T. W. Ebbesen, and L. Kuipers, Rev. Mod. Phys. 82, 729 (2010). 